\title{
Retinal pathology in the Kearns-Sayre syndrome
}

\author{
N M McKECHNIE, ' M KING, ${ }^{2}$ AND W R LEE' \\ From the ${ }^{1}$ Departments of Pathology and Ophthalmology, University of Glasgow, Western Infirmary, \\ Glasgow and the ${ }^{2}$ Royal Hospital for Sick Children, Glasgow
}

\begin{abstract}
SUMMARY Examination of the retinal tissues obtained at necropsy from a 14-year-old boy with Kearns-Sayre syndrome showed marked photoreceptor and pigment epithelial cell loss in the retinal periphery and around the optic nerve head. Electron microscopy of surviving retinal pigment epithelial (RPE) cells indicated a loss of apical microvilli and basal infoldings. The RPE was unusually devoid of melanosomes and showed no evidence of phagocytosis of photoreceptor debris. The cytoplasm of the RPE contained numerous, often enlarged, mitochondria. These structural changes suggested that a breakdown in the energy dependent interrelationships between the RPE and the photoreceptor layer was responsible for the outer retinal degeneration. The finding of numerous macrophages in the subretinal space suggests a secondary inflammatory component in the retinal degeneration.
\end{abstract}

This paper presents the histopathological and ultrastructural findings in the well preserved retinal tissues of a 14-year-old boy suffering from Kearns disease of infantile onset or Kearns-Sayre syndrome. Two years prior to death the boy was night blind and had an obvious pigmentary retinopathy. The basic abnormality in this rare systemic disorder is thought to be a disturbance in mitochondrial morphology and function that is assumed to cause a breakdown in photoreceptor maintenance and phagocytosis. Previous morphological records of the disease in the retina are confined to six studies with the use of light microscopy ${ }^{1-6}$ and two investigations by electron microscopy. ${ }^{7-8}$ The discrepancies present in the previous morphological descriptions suggest that the syndrome is more complex than first thought. A detailed morphological study was therefore justified, and our findings are discussed in relation to the previously reported material.

\section{Case report}

A boy, the fourth child of healthy, non-consanguineous Scottish parents, was born at term after an uneventful pregnancy, birth weight $3 \mathrm{~kg}$, length 52 $\mathrm{cm}$, head circumference $35 \mathrm{~cm}$. There were no neonatal problems, but during the first year failure to thrive and psychomotor delay were noted.

Correspondence to Professor W R Lane, Department of Ophthalmology, Western Infirmary, Glasgow G11 6NT.
At 13 months the child was admitted to hospital with pallor and gingival bleeding. Peripheral blood examination showed pancytopenia ( $\mathrm{Hb} 5.34 \mathrm{~g} / \mathrm{dl}$, leucocytes $3 \cdot 4 \times 10^{9} / 1,5 \%$ polymorphs, platelet count $23 \times 10^{9} / 1$ ). Bone marrow aspirate showed marked hypoplasia of all cell lines. The patient was transfused with red cell concentrate and made a good recovery. The hypoplasia was regarded as transitory, and no further haematological abnormality was noted at follow-up.

He walked at 3 years, spoke sentences at 5 , and attended a special school. At 7 years the following features were noted: short stature, mental retardation (IQ 64), cerebellar ataxia, dysarthria, bilateral ptosis (Fig. 1), progressive external ophthalmoplegia, decreased visual acuity, atrophic maculae, punctate cataracts, sensorineural deafness, generalised muscle weakness, diminished tendon reflexes, and hyperventilation.

Investigations showed partial growth hormone deficiency, metabolic acidosis, raised serum pyruvate and lactate, and restrictive lung disease. Chromosome karyotype was normal. A muscle biopsy (quadriceps) showed atrophic muscle fibres with some ragged red fibres, and a diagnosis of KearnsSayre syndrome was made.

At 12 years a cardiac pacemaker was inserted for Stokes-Adams attacks associated with heart block. Tetany, due to primary hypoparathyroidism, was controlled with hydroxycholecalciferal. 


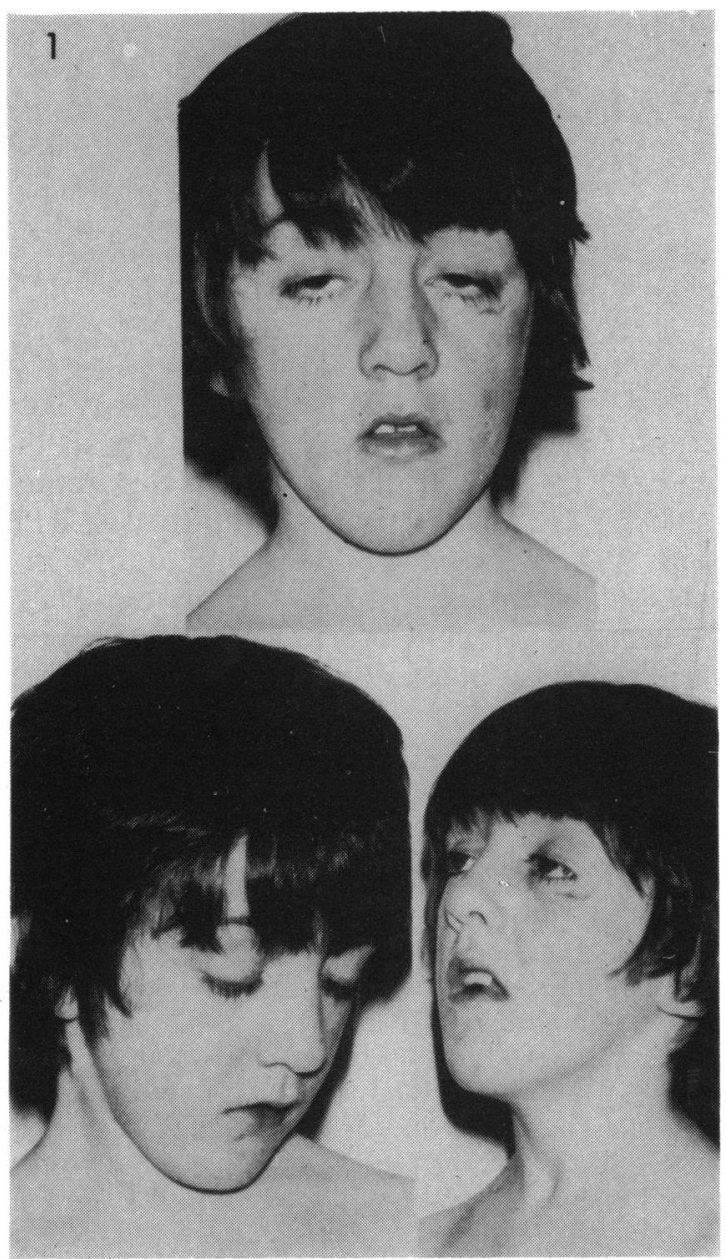

Fig. 1 The patient one year before death.

The boy suffered intractable cardiac failure at age 14 and died after three days at 1400 hours.

\section{Materials and methods}

The left and right eyes were obtained at necropsy. Phosphate buffered glutaraldehyde $(2 \%)$ was injected into the vitreous immediately post mortem. The enucleated eyes were fixed in formal saline.

Left eye. After gross examination the left eye was processed conventionally for paraffin histology. Serial sections were cut in the horizontal plane through the pupil, the macula, and the optic nerve head.

Right eye. Twenty four tissue blocks were taken from the locations shown in Fig. 2. In addition a small central area of retinal tissue was taken for examination by scanning electron microscopy. After dissec-

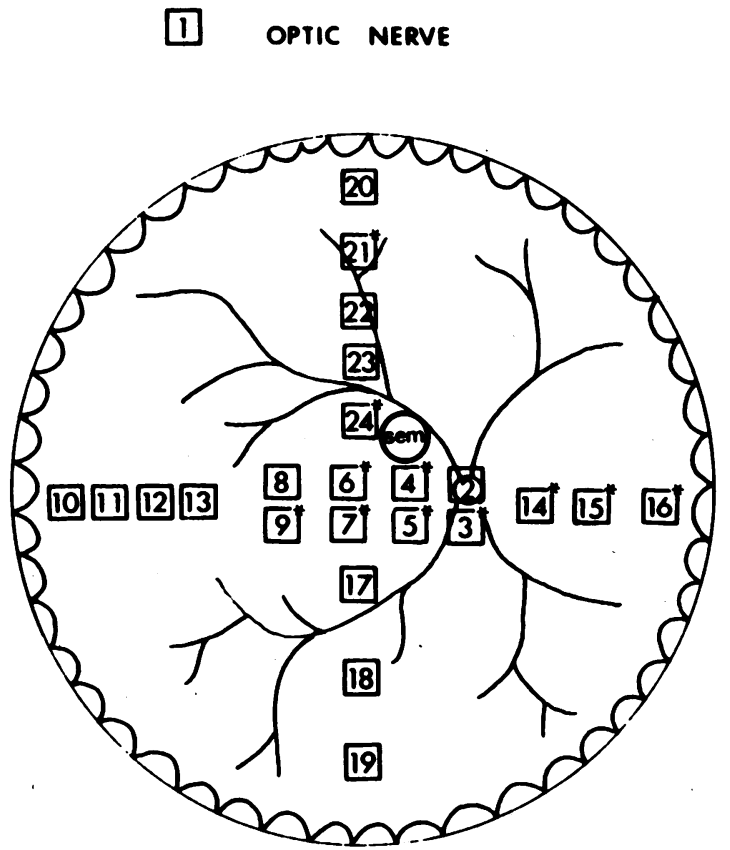

Fig. 2 Locations in the right eye of tissue blocks taken for investigation. Block numbers correspond to those in Table 1. *Indicates that photoreceptors were found in that block.

tion the tissue blocks were additionally fixed in $2 \%$ glutaraldehyde buffered with $0 \cdot 1 \mathrm{M}$ sodium cacodylate $(\mathrm{pH} 7 \cdot 2)$. After secondary fixation in $1 \%$ osmium tetroxide in $0.1 \mathrm{M}$ sodium cacodylate buffer the tissue was dehydrated through a graded series of acetone solutions and embedded in Transmit resin prior to examination in a Phillips 301 electron microscope. The tissue for scanning electron microscopy was taken from the perimacular area (see Fig. 2) and was prepared by the critical point drying method for examination in a Jeol SEM (2B).

Semithin sections were cut from all the designated blocks. Blocks that showed interesting or unusual features were recut for electron microscopy.

\section{Results}

The left and right eyes showed similar macroscopic appearances. In addition the histology in the paraffin sections of the left eye was found to correlate well with the findings obtained in the right eye by light and electron microscopy.

Left eye (macroscopic). The anterior part of the eye was of normal appearance. At the retinal periphery there was some fine pigmentary speckling, while towards the posterior pole there was gross pallor and 


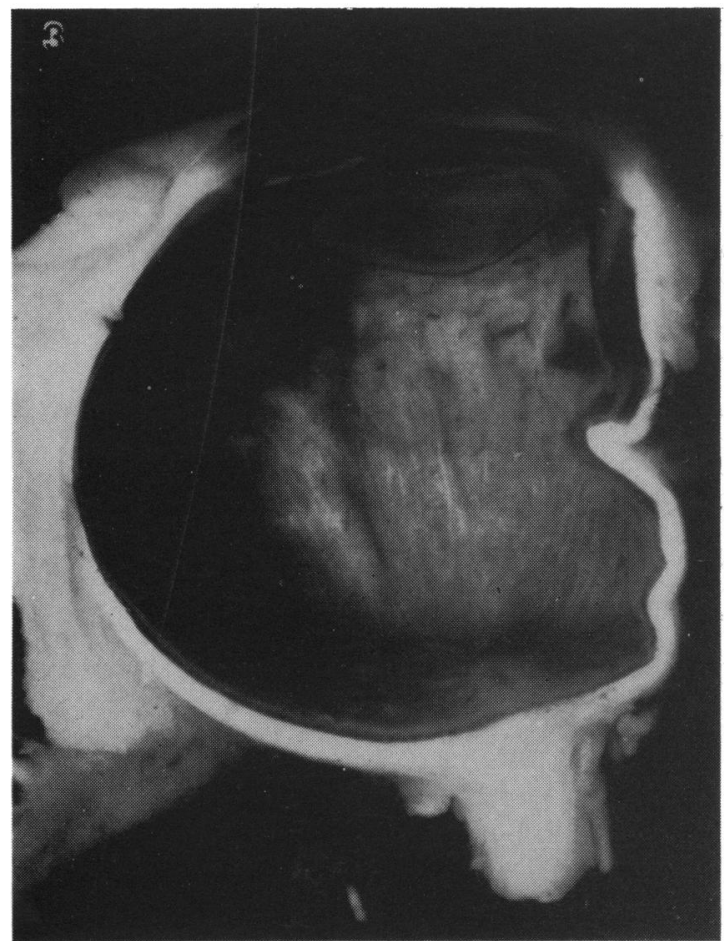

Fig. 3 Macroscopic photograph of the left eye. Note the slight bone spicule pigmentation in the periphery and the 'salt and pepper' appearance of the more posterior fundus.

loss of pigmentation. The optic disc was white. The retinal vessels, the choroid, and the sclera were unremarkable (Fig. 3).

Left eye (microscopic). The most significant abnormalities were observed in the outer retina. Photoreceptor cells were absent except at the posterior pole. There was, however, a peripapillary region of cell loss (Fig. 4a). In areas of photoreceptor survival retinal pigment epithelial (RPE) cells were also present. These regions of surviving pigment epithelial photoreceptor complex appeared to form discrete islands of cells that were more common toward the posterior pole (Fig. 4a, b, c, d, e). At the edge of these areas the outer limiting membrane appeared to bend inwards to fuse with the RPE and to enclose residual photoreceptors and macrophages in the subretinal space. Large pigment laden cells were often seen within such areas of preservation (Fig. 4b). Posteriorly the surviving RPE appeared to be attenuated and depleted of melanin (Fig. 4c, d, e). Where the RPE monolayer was absent, gliotic outer retina appeared to fuse directly with Bruch's membrane (Fig. 4f). In the peripheral retina there was complete atrophy of the outer retina with occasional migration of isolated pigment containing cells into the residual atrophic retinal tissue (Fig. 4g). Fluorescence microscopy failed on unstained sections to detect any lipofuscin granules in the pigment epithelium or the exogenous cells in the retina.

The optic disc and the optic nerve contained numerous axons, but in absolute numbers the impression was that there were fewer than in normal tissue of this age group. Axons were absent from the peripheral bundles of the optic nerve, though myelination appeared to be normal in the central part of the optic nerve.

Right eye (light microscopy, transmission, and scanning electron microscopy). A summary of the light microscopic findings is given in Table 1.

At the ora serrata there was marked hyperplasia of the RPE (Fig. 5a, b). Occasionally an epiretinal membrane was present (Fig. 5b). Beneath these areas of RPE hyperplasia fibrovascular proliferation was found (Fig. 5a). Blood vessels were also found beneath the pigmented epithelium but above Bruch's membrane in the region of the pars plana (Fig. 5c). At the far retinal periphery the outer nuclear layer was absent, though a few isolated pigment epithelial cells were in contact with expanded Muller cell cytoplasm (Fig. 5d). In the paramacular area the isolated regions of photoreceptors and RPE preservation were more profitably studied in semithin sections and were found to vary in size and morphological characteristics. In some segments there were recognisable monolayers of RPE with numerous photoreceptors (Fig. 5e), while in other areas circular spaces surrounded residual photoreceptors, and a few RPE cells persisted (Fig. 5f). Frequently these enclosed regions of the subretinal space contained large pigment laden cells (Fig. $5 \mathrm{e}$ and $5 \mathrm{~g}$ ). In the macular region the outer retina was of more normal appearance, though there appeared to be a marked reduction in the thickness of the outer nuclear layer and attenuation of the RPE cell layer (Fig. 6a). As in other regions of surviving photoreceptors cells of macrophagic character were often seen within the subretinal space (Fig. 6b).

Electron microscopy of the regions of surviving photoreceptor/RPE cell complex confirmed the presence of macrophages within the subretinal space (Figs. 7 and 8a). These macrophagic cells contained both photoreceptor cell outer segment debris and spindle shaped melanin granules that appeared to be of pigment epithelial origin.

The surviving RPE was of abnormal appearance. It lacked both apical villi and basal infoldings (Figs. 7, $8 \mathrm{a}, \mathrm{b}$ ) and contained no phagosomes or lipofuscin. However, a striking feature of the RPE was the presence throughout the cytoplasm of large numbers of mitochondria (Figs. 7, 8a, b, 9a, b). The distribution was quite different from the normal in which 


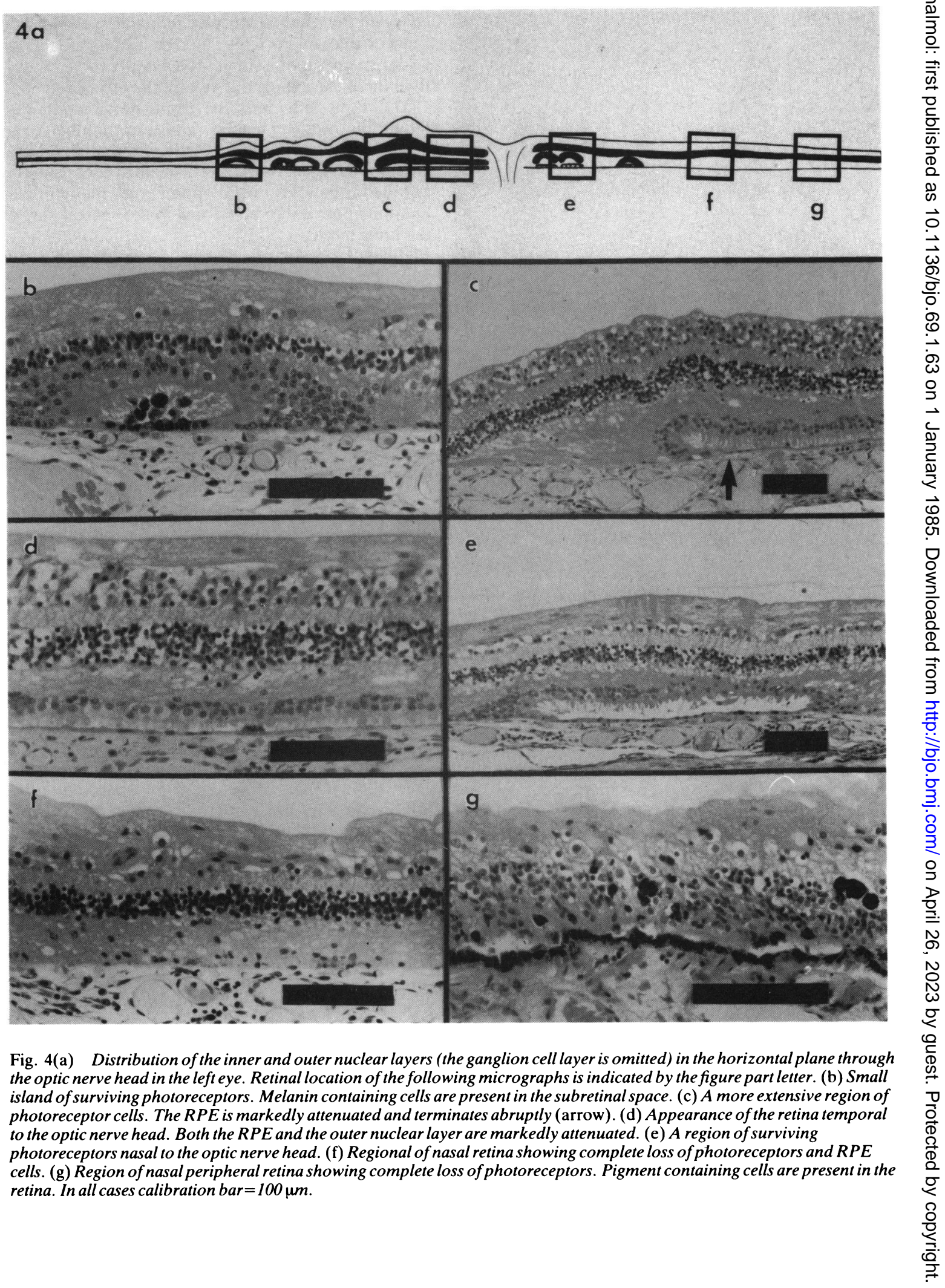


Table 1 Summary of light microscopic findings

\begin{tabular}{llll}
\hline $\begin{array}{l}\text { Tissue } \\
\text { block } \\
\text { No }\end{array}$ & Asstologyment of retinal structure & Comments \\
\cline { 2 - 4 } & RPE Cones $\ddagger$ Rods $\ddagger \quad \begin{array}{l}\text { Pigmented } \\
\text { macrophages } \|\end{array}$
\end{tabular}

\begin{tabular}{|c|c|c|c|c|c|c|}
\hline 1 & Normal & - & - & - & - & - \\
\hline 2 & Abnormal & Absent & Absent & Absent & Absent & Retina absent, only nerve fibre layer present \\
\hline 3 & Abnormal & Patchy & Present* & Present ${ }^{*}$ & Present SR & $\begin{array}{l}\text { Numerous macrophages associated with photoreceptor outer } \\
\text { segments }\end{array}$ \\
\hline 4 & Abnormal & Patchy & Present $\dagger$ & Present $\dagger$ & Present SR & Macrophages numerous $\|$ \\
\hline 5 & Abnormal & Patchy & Present* & Present* & Present SR & - \\
\hline 6 & Abnormal & Patchy & Present $\dagger$ & Present $\dagger$ & Present SR & - \\
\hline 7 & Abnormal & Patchy & Present* & Present ${ }^{*}$ & Present SR & - \\
\hline 8 & Abnormal & Absent & Absent & Absent & Present & 一 \\
\hline 9 & Abnormal & Patchy & Present $\dagger$ & Present ${ }^{*}$ & Present SR NR & Small enclosed islands of photoreceptors $\llbracket$ \\
\hline 10 & Abnormal & Present & Absent & Absent & Present NR & Hyperplasia of RPE at ora serrata \\
\hline 11 & Abnormal & Patchy & Absent & Absent & Present NR & -1 \\
\hline 12 & Abnormal & Patchy & Absent & Absent & Present SR NR & - \\
\hline 13 & Abnormal & Patchy & Absent & Absent & Present NR & - \\
\hline 14 & Abnormal & Present & Present $\dagger$ & Present§ & Present SR & 一 \\
\hline 15 & Abnormal & Absent & Present & Present $\dagger$ & Present NR & Small enclosed islands of photoreceptors $\Uparrow$ \\
\hline 16 & Abnormal & Patchy & Present & Present & Present SR NR & - \\
\hline 17 & Abnormal & Absent & Absent & Absent & Present NR & - \\
\hline 18 & Abnormal & Patchy & Absent & Absent & Present NR & 一 \\
\hline 19 & Abnormal & Present & Absent & Absent & Absent & Capillaries below pigmented epithelium $\rrbracket$ \\
\hline 20 & Abnormal & Absent & Absent & Absent & Present NR & - \\
\hline 21 & Abnormal & Patchy & Present* & Present* & Present SR NR & Small island of photoreceptors and macrophages $\mathbb{1}$ \\
\hline 22 & Abnormal & Absent & Absent & Absent & Present NR & - \\
\hline 23 & Abnormal & Absent & Absent & Absent & Present NR & - \\
\hline 24 & Abnormal & Present & Present* & Present ${ }^{*}$ & Present SR & - \\
\hline
\end{tabular}

Rods and cones. Present $=$ Cell body present no inner or outer segments seen. Present ${ }^{*}=$ Cell body and inner segment seen. Present $\dagger=$ Cell body and both inner and outer segments seen.

Pigmented macrophages. $\mathrm{NR}=$ Macrophages in neural retina. $\mathrm{SR}=$ Macrophages in subretinal space.

$\ddagger$ Receptor cells found only in association with surviving RPE cells.

$\S$ Very unusual circle of photoreceptors.

॥Macrophages described as in neural retina when RPE and photoreceptors completely absent, even though they may be in contact with Bruch's membrane.

IFeature shown as illustration.

mitochondria are confined to the basal part of the cytoplasm. These mitochondria were in some areas very well preserved and contained plate like cristae. Bizarre mitochondria, as described in muscle fibres in some forms of the Kearns-Sayre syndrome, were not observed within the RPE monolayer, though large and irregularly shaped mitochondria were prominent (Fig. 9a, b).

Though it would have been desirable to quantify the apparent increase in the number of mitochondria per cell, this was not possible for two reasons. First, normal control material is not available. Secondly, the extreme variation in RPE cell size in the pathological material would have made a quantitative analysis extremely difficult and time consuming. Normal adult RPE cells have an approximate width of $20 \mu \mathrm{m}$ and are $10 \mu \mathrm{m}$ high. The RPE cells in our material were 7-10 $\mu \mathrm{m}$ high but were highly irregular in shape. Some of them were spindle shaped and measured more than $60 \mu \mathrm{m}$ in their greatest

Fig. 5 Composite figure showing the appearance of various retinal areas as seen by light microscopy of plastic embedded tissue. (a) (Table 1, block 10). Region of RPE hyperplasia at the ora serrata. Note the blood vessels between Bruch's membrane and the pigmented epithelium. Bar $=40 \mu \mathrm{m}$. (b) (Table 1, block 10). A more peripheral region of the same block as shown above. An epiretinal membrane is present. Bar $=40 \mu \mathrm{m}$. (c) (Table 1, block 19). Pars plana adjacent to the ora serrata. There are prominent capillaries under the pigmented epithelium. Bar $=20 \mu \mathrm{m}$. (d) (Table 1, block 11). A region of complete photoreceptor cell loss. Some RPE cells are present as well as a few pigment containing cells. Bar $=40 \mu m$. (e) (Table 1, block 9). An island of surviving photoreceptor cells in the posterior retina. Pigment containing cells are prominent in the subretinal space. Bar $=40 \mu \mathrm{m}$. (f) (Table 1, block 15). Unusual circular structure formed by curving round and down of the outer limiting membrane. Bar $=20 \mu \mathrm{m}$. (g) (Table 1, block 21). A region of surviving photoreceptors in the retinal periphery. The small enclosed region of subretinal space contains numerous pigment laden cells. The RPE, while typically amelanotic, contains melanosomes in this area. Bar $=10 \mu \mathrm{m}$. 


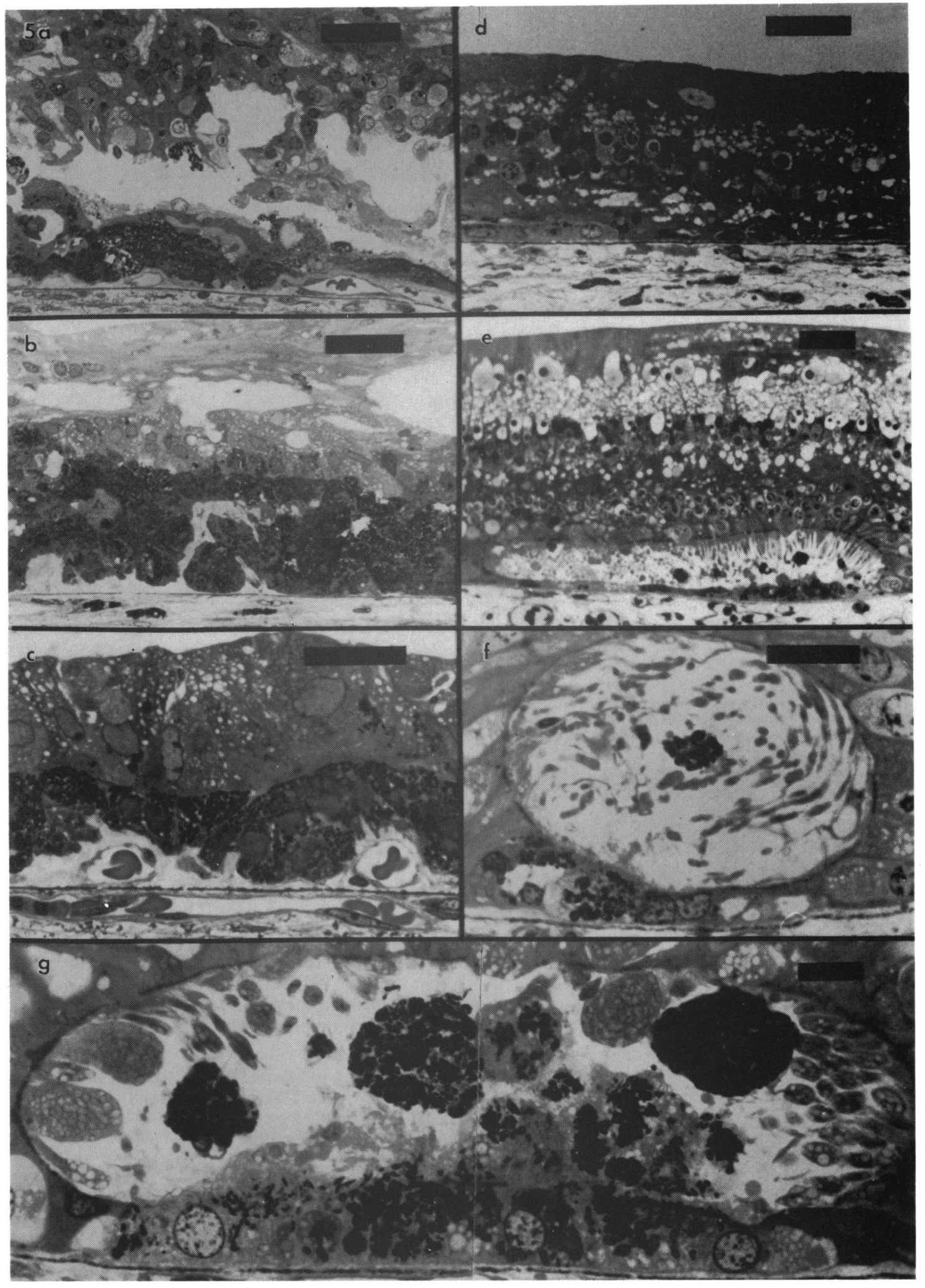




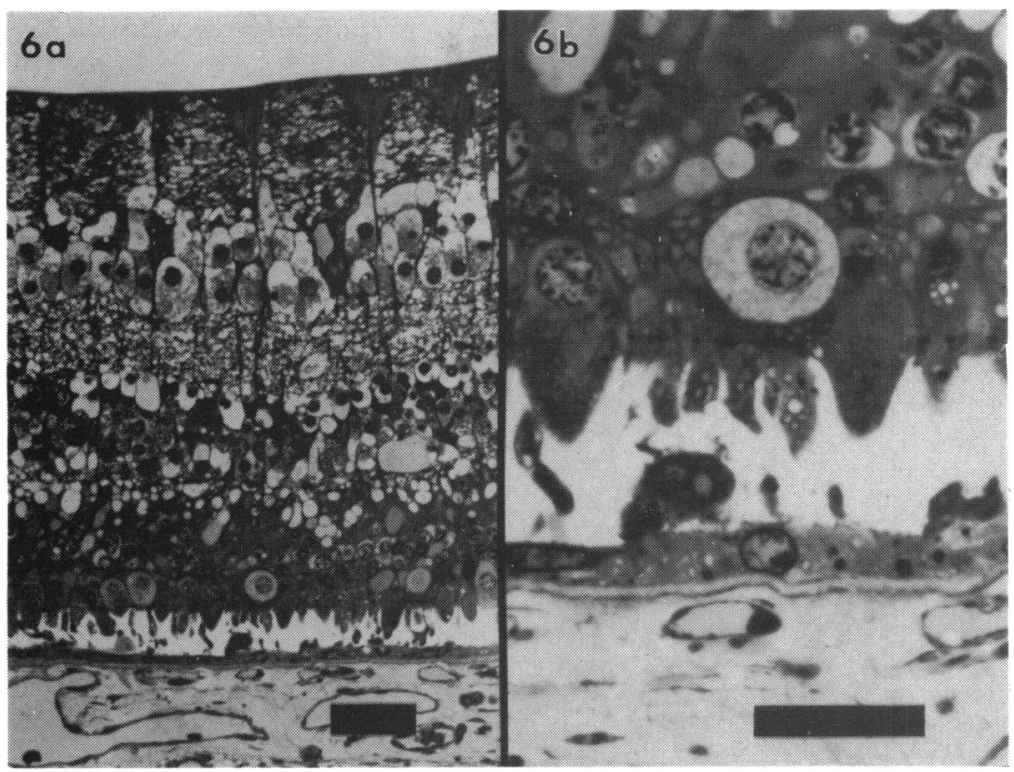

Fig. 6 (Table 1, block 4). (a) A region of central retina where there is a marked reduction in the number of photoreceptor cells. Bar $=40 \mu \mathrm{m}$. (b) Same area at higher magnification showing attentuation of the choriocapillaris and a cell of macrophagic appearance in the subretinal space. Bar $=20 \mu \mathrm{m}$.

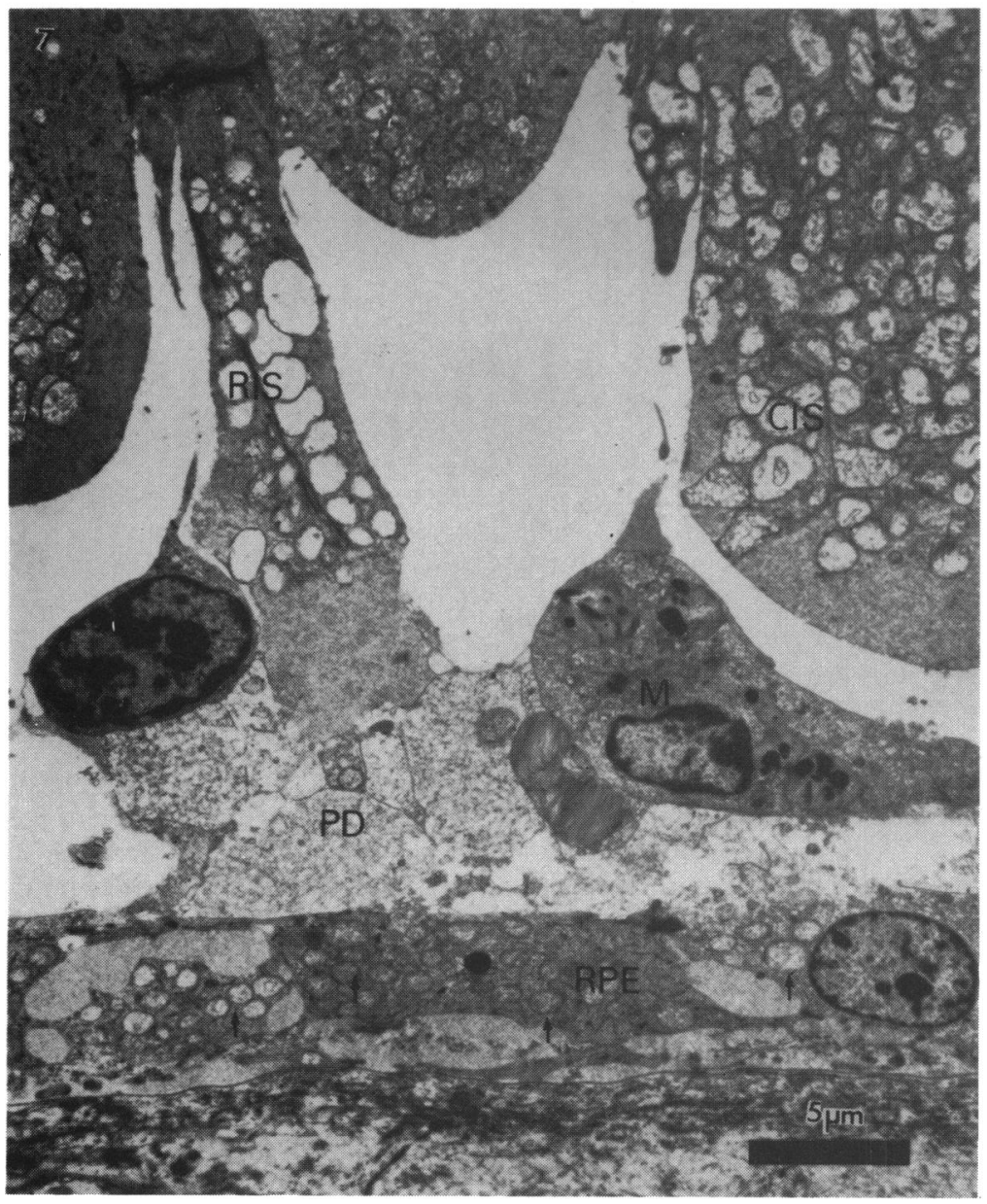

Fig. 7 Electron micrograph showing the appearance of the photoreceptors and pigment epithelium. $\mathrm{M}=$ macrophage, $\mathrm{CIS}=$ coner inner segment, RIS $=$ rod inner segment, $\mathrm{RPE}=$ retinal pigment epithelium, $\mathrm{PD}=$ photoreceptor debris, Mitochondria, shown by arrows. 
Fig. 8 Electron micrographs showing the RPE (a) with pigment containing macrophages on its apical surface, and (b) the accumulation of outer segment material. Note the absence of apical microvilli and basal infoldings. $\mathrm{ROS}=$ rod outer segment, $\mathrm{M}=$ macrophage, $\mathrm{RPE}=$ retinal pigment epithelium, $\mathrm{PD}=$ photoreceptor debris, Mitochondria, shown by arrows.

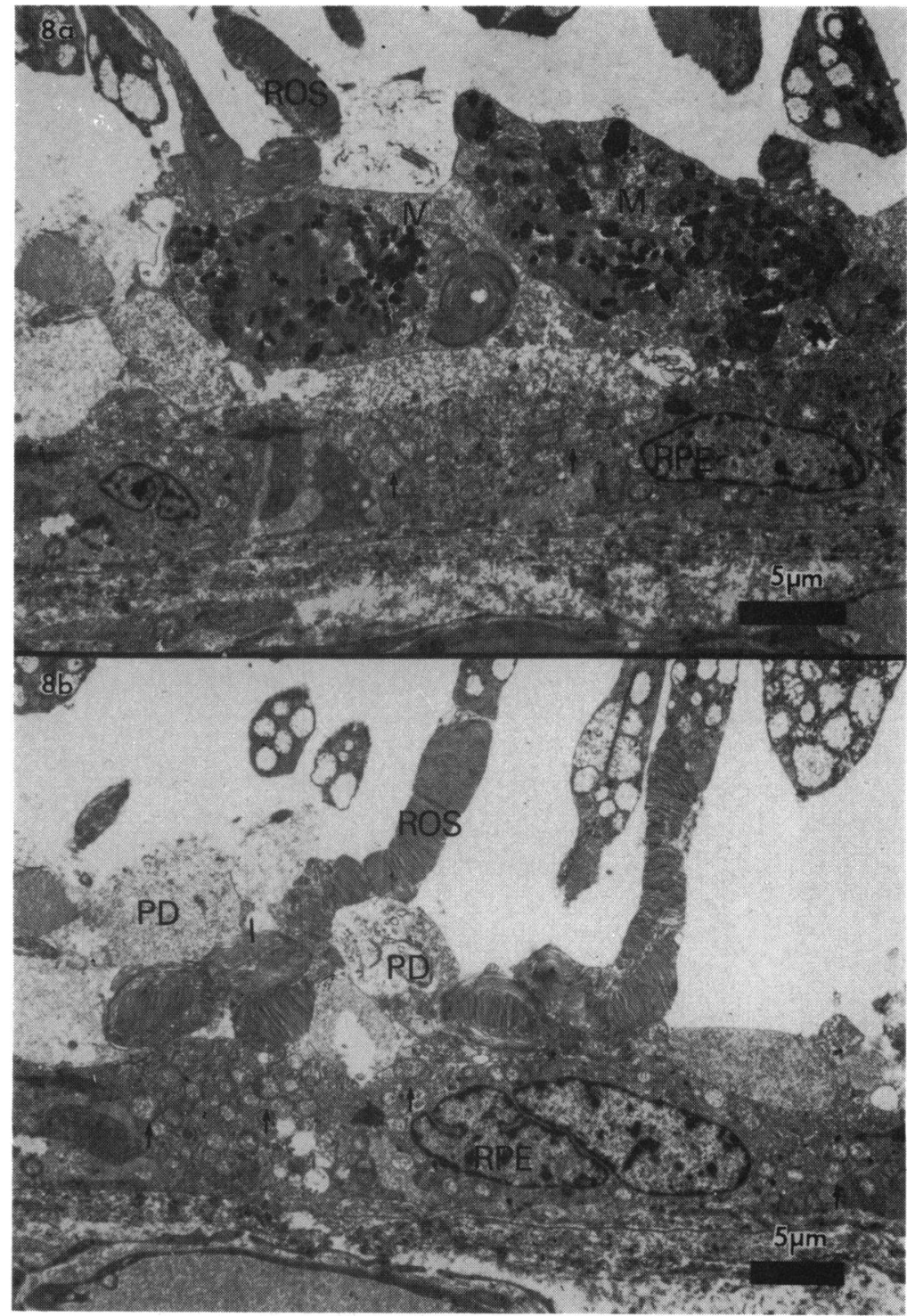

dimension. This difference in cell shape effectively precluded meaningful quantitation of the numbers of mitochondria per cell.

In some areas there was an accumulation of photoreceptor debris on the apical surface of the RPE cells (Fig. 8b). It was not possible to be sure in this material if the membrane bound debris represented either dislocated tips or fragments of outer segments. The important finding was that, although the rod outer segment debris was often in intimate contact with the RPE, there was no evidence of phagocytosis (Figs. 8b, 9a, b).

In all the surviving RPE cells the apical junctional complex was present (Fig. 9a, b), though some cells showed a marked elongation and convolution of the lateral cell membrane (Fig. 9b). An absence of proteinaceous material between the surviving photoreceptor outer segments suggested that in areas of surviving RPE the outer retina blood barrier was intact. Sophisticated study of tight junctions (for example, by freeze fracture) was not possible in this material. Scanning electron microscopy of the inner surface of the RPE surface suggested that the RPE cells were highly irregular in shape (Fig. 10a). This irregularity of shape probably accounted for the elongation of the lateral cell membrane seen by 

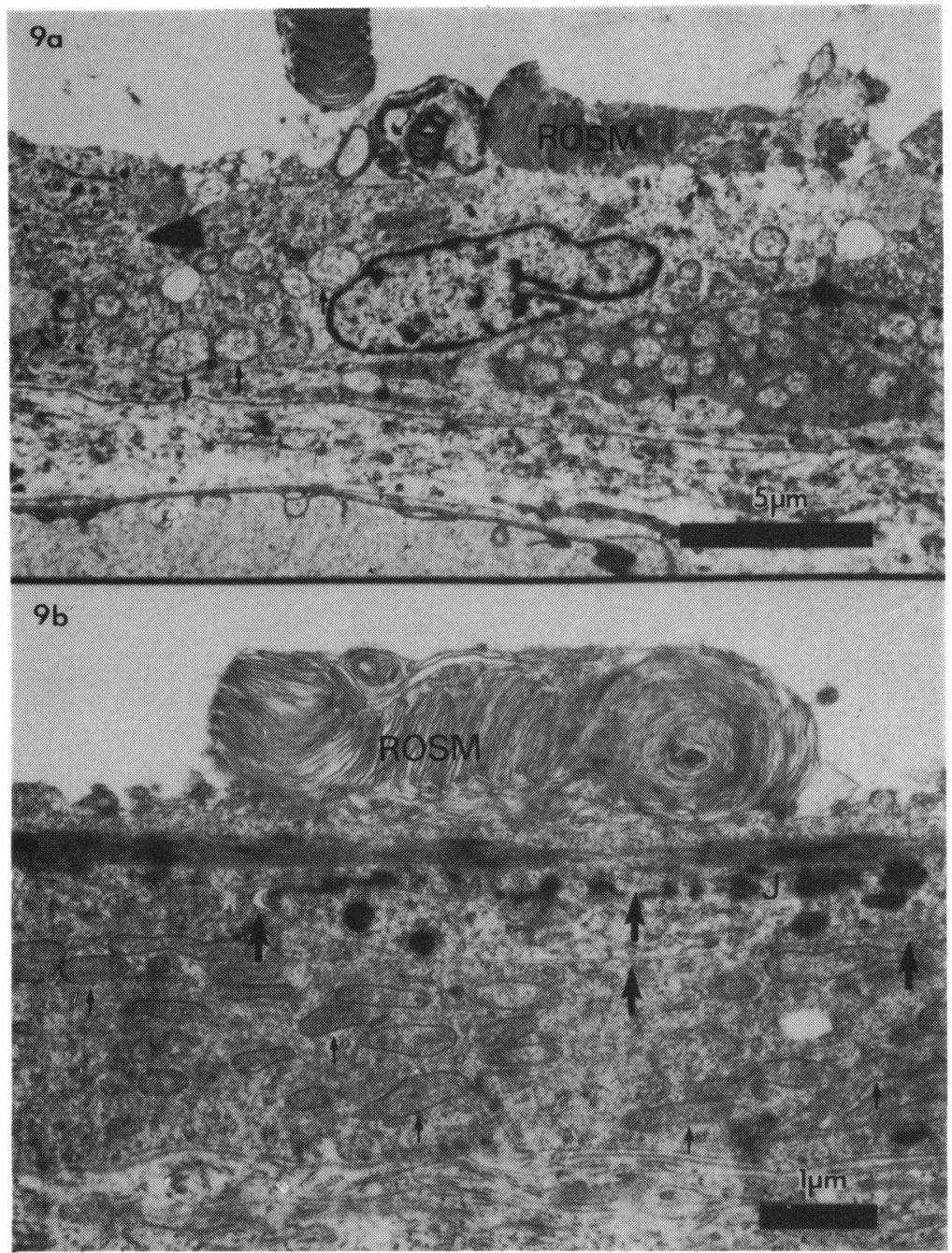

Fig. 9(a) Electron micrograph of an RPE cell with rod outer segment material in contact with its apical surface. Thee is no indication of phagocytosis. (b) Similar area as above but at higher magnification. Note the numerous mitochondria and the elongated lateral cell membrane. $\mathrm{ROSM}=$ rod outer segment material, $\mathrm{J}=$ junctional complex. Mitochondria shown by arrows. Larger arrows show lateral cell membrane. transmission electron microscopy. By scanning electron microscopy it was frequently difficult to determine if the subretinal cells were irregular shaped intrinsic cells of the RPE monolayer or macrophagic cells in intimate contact with the surface of the underlying monolayer. Transmission electron microscopy of similar areas to those shown in Fig. 10a suggested that some of the surface cells were extrinsic to the monolayer (Fig. 10b).

Frequently cells of unknown origin were seen between the lateral cell membranes of the RPE cells (Figs. 10b, 11a, b). These cells had an appearance compatible with that of differentiating mononuclear leucocytes and were considered to be precursors of the subretinal macrophages that contained melanophagosomes and outer segment debris (Figs. 7, 8a, 10b).

\section{Discussion}

There is some controversy as to the definition of Kearns-Sayre syndrome. Drachman" coined the term 'ophthalmoplegia plus' to encompass the variety of neural, cardiac, and retinal disorders that may occur in conjunction with ophthalmoplegia. However, there is a consistent trend in those cases that begin in the first or second decades of life, and this is characterised by ophthalmoplegia, atypical pigmentary degenerations of the retina, and heart block. This is the clinical combination originally described by Kearns and Sayre.' Some authors, however, have noted other associated features such as spongy degeneration of the brain, ${ }^{4}$ raised cerebrospinal fluid protein levels, ${ }^{10}$ and hypoparathyroidism. ${ }^{6}$ Hypoparathyroidism was present in the patient described here. 
Fig. 10(a) Scanning electron micrograph showing the apical surface of the RPE and a torn edge of the retina. Inflammatory cells are present on the surface of a highly irregular layer of RPE cells. (b) $A$ similar area as seen by transmission electron microscopy. Note the small cell between the RPE cells (arrow). $\mathrm{CIS}=$ cone inner segment, $\mathrm{M}=$ macrophage, $\mathrm{RPE}=$ retinal pigment epithelium.

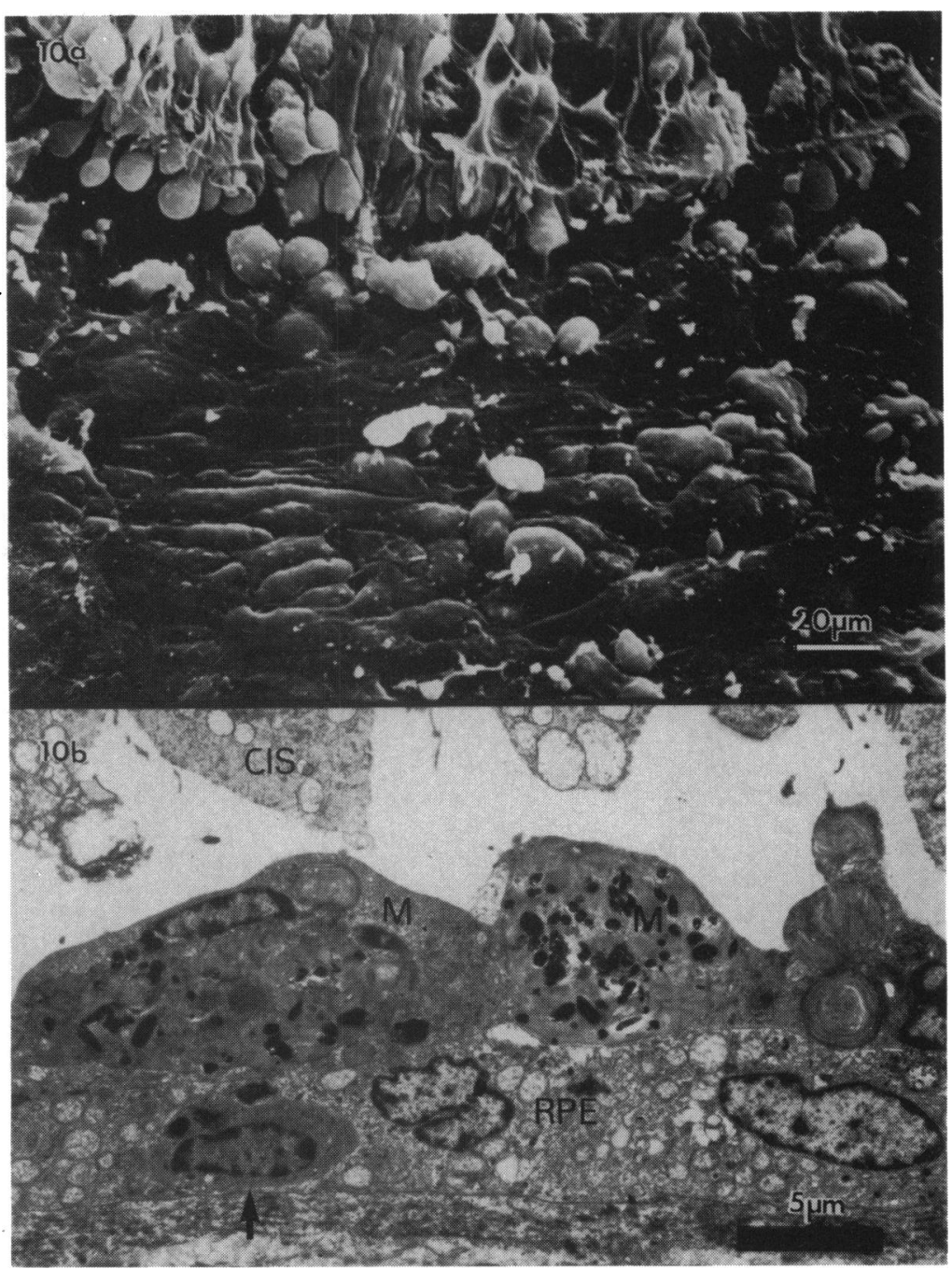

In agreement with Berenberg et al., ${ }^{10}$ Bastiaensen et al., ${ }^{11}$ and François ${ }^{12}$ we feel that the term KearnsSayre syndrome should be restricted to the disease of infantile onset-a multisystemic neuromuscular disease with chronic progressive external ophthalmoplegia, obligate cardiac and retinal signs, and a high protein content of the cerebrospinal fluid. It should be added that Bastiaensen et al." have also described a juvenile form and an adult form characterised by onset in the third decade of life or later. These juvenile and adult forms are probably best classified as Kearns disease. ${ }^{12}$ The course of all the subgroups is variable, but the severity appears to be related to the age of onset. The infantile form, the Kearns-Sayre syndrome, has the worst prognosis.

The retinopathy of Kearns-Sayre syndrome or Kearns disease is usually termed atypical to distin- guish it from the more characteristic pigmentary retinopathy of the retinitis pigmentosa group of diseases. The retinal degeneration in Kearns-Sayre syndrome often occurs centrally and without a conspicuous pigmentation of the peripheral retina. ${ }^{13}$ The fundus is more typically described as having a 'salt and pepper' or 'motheaten appearance. ${ }^{13}$

To the best of our knowledge there are eight reports in the literature in which the retina of KearnsSayre syndrome has been examined histologically, five by conventional wax histology ${ }^{1-6}$ (Kearns reporting the same case twice ${ }^{12}$ ) and two by electron microscopy. ${ }^{78}$ The reports that mention or illustrate the retinal histology differ considerably. Those of Kearns and Sayre ${ }^{1}$ and Kearns ${ }^{2}$ describe a local absence of rods and cones with an almost complete absence of the pigment epithelium. The outer 


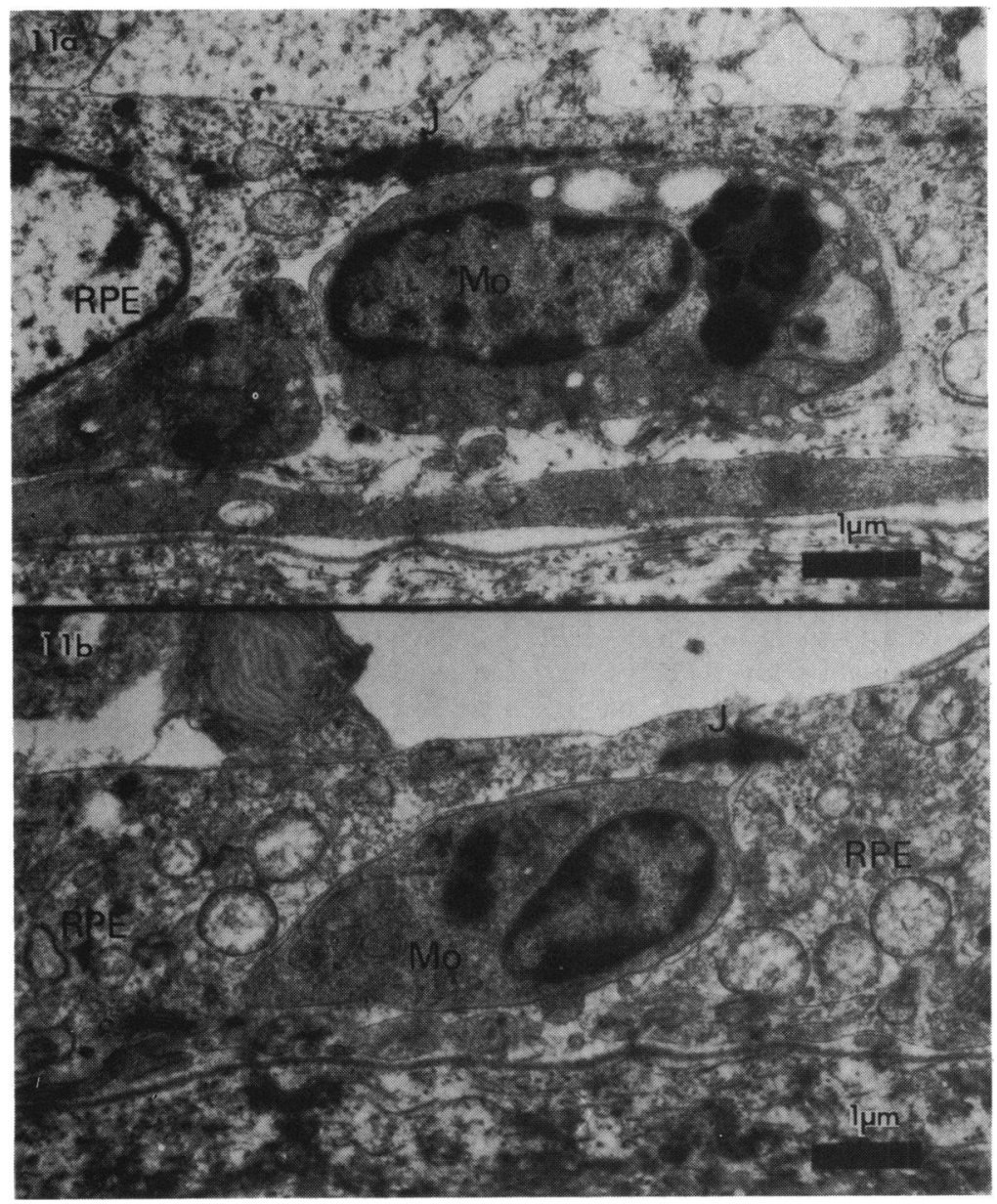

Fig. 11(a),(b) Electron micrographs of the small cells seen between the lateral cell membranes of RPE cells. These cells are thought to be monocytes. $\mathrm{Mo}=$ monocyte, $\mathrm{RPE}=$ retinal pigment epithelium, $\mathrm{J}=$ junctional complex.

nuclear layer was absent in areas, and much of the retina was gliotic. An essentially similar description of the retinal morphology was given by Castaigne and coworkers. ${ }^{5}$ Horwitz and Roessmann ${ }^{6}$ reported only a few rods at the posterior pole, while the rod and cone layer was absent elsewhere. The findings in these studies are quite similar to the findings in this study. In contrast the study of Jager et al. ${ }^{3}$ reported no histological abnormality of the retina, and that of Daroff $e t ~ a l .{ }^{4}$ mentions only a degeneration of rods and cones.

The report of Newell and Polascik ${ }^{7}$ describes the light and electron microscopic findings, both transmission and scanning, in the post mortem eyes of a 19-year-old. They reported the presence of enlarged mitochondria in the RPE (similar to our findings in this study), the receptor cell inner segments, and the ciliary body. They also reported the presence of large macrophages within the subretinal space. As in this study these cells were reported to contain receptor cell outer segment debris. However, they also reported ingestion of receptor cell outer segment material by the RPE. This was not observed in the present case. The only other electron microscopic study is that of Eagle et al., ${ }^{8}$ who described the findings in the eyes of a 52-year-old man. Their findings differ from those in the present case in some important respects. The photoreceptor and RPE cell loss was most marked toward the posterior pole in their material. In the present study the photoreceptor and RPE cell loss was most marked in the retinal periphery, though some loss of photoreceptors and RPE cells was evident around the optic nerve head. Eagle $e t$ al. ${ }^{8}$ also reported a patchy depigmentation of the RPE with some cells containing large granules or aggregates of pigment granules. They did not report any mitochondrial abnormalities. In our material the majority of the RPE cells were devoid of melanosomes but did contain numerous, often enlarged, mitochondria. The differences between these two 
studies can be attributed to the age difference and a more rapid development of the disease, but the possibility of different pathogenetic processes within the spectrum of the Kearns-Sayre syndrome must also be accepted.

Abnormal mitochondria have been reported in patients with chronic progressive external ophthalmoplegia. ${ }^{14}$ In the study of Mitsumoto et al..$^{14}$ the patients showing ultrastructural mitochondrial abnormalities all had characteristic ragged red fibres in biopsies of muscle. These authors found that the ultrastructural appearance of mitochondria obtained by muscle biopsy did not correlate with measured rates of respiratory function in isolated mitochondria. However, they did record an inverse relationship between morphometric mitochondrial fraction (numbers or area of mitochondria in electron micrographs) and the respiratory rate of the isolated mitochondria. These authors ${ }^{14}$ suggest the possibility of mitochondrial proliferation in compensation for reduced efficiency. It is possible that such a compensating mechanism was operating in the RPE we studied and was responsible for the large numbers of mitochondria observed throughout the cell cytoplasm. Other authors have shown reductions in cytochrome c oxidase activity, reduced concentrations of cytochrome $\mathrm{aa}_{3}$, and a decreased ratio of cytochrome oxidase/succinate cytochrome c reductase and defects in pyruvate metabolism in patients with chronic external ophthalmoplegia, ${ }^{15}{ }^{16}$ and defects in the mitochondrial respiratory chain located at $\mathrm{NADH} \mathrm{CoQ}$ reductase and at cytochrome $\mathrm{b}$ in patients with mitochondrial encephalomyopathies. ${ }^{17}$ These types of defect would all produce 'inefficient' mitochondria and again emphasise the possibilities of pathogenetic variation in the spectrum of the syndrome. Transmission electron microscopy studies of other tissues in patients with ophthalmoplegia plus and Kearns-Sayre syndrome have shown abnormal mitochondria in the cerebellum, ${ }^{18}{ }^{19}$ myocardium, ${ }^{20}$ sweat glands, ${ }^{21}$ and liver. ${ }^{22}$

The pathogenesis of Kearns-Sayre syndrome in the outer retina is difficult to establish from a single case. However, the gradation of damage, severest in the periphery and moderate at the posterior pole, probably illustrates the development of the degeneration. It could be assumed that the outer retinal degeneration seen in the later stages (peripheral retina) may be potentiated by the macrophages present within the outer retina. This point is of some interest as, regardless of primary pathology, an inflammatory process in the outer retina appears to be exacerbating the degree of outer retinal damage. This secondary inflammation of the outer retina may be of considerable consequence in retinitis pigmentosa ${ }^{23}$ and other conditions where the outer retina is affected, such as uveitis and toxoplasmosis. ${ }^{24}$ The potential of an autoimmune component is witnessed by the increasing interest in circulating anti-s antibodies in a variety of retinal conditions. ${ }^{24}$

However, the primary cause of the retinal degeneration in Kearns-Sayre syndrome remains obscure, though some speculation is possible. The role of defective RPE transport systems in the development of retinal degeneration has been stressed by Bird and Marshall. ${ }^{25}$ We can now assume that the RPE is normally a very metabolically active cell monolayer. The numerous mitochondria adjacent to the basal infoldings and the numerous apical microvilli indicate that the RPE has a variety of transport systems, many of which must be energy dependent. Therefore any reduction in the cell's energy producing capacity will compromise the transport systems and phagocytosis, which is known to be an energy dependent phenomenon..$^{26}$ Therefore it is also likely to be reduced in an energy deficient RPE cell. We may then assume that the first stage in the receptor cell damage is due to an insufficient transport of metabolites via the RPE. The observed accumulation of photoreceptor debris in the subretinal space is interpreted as a consequence of impaired phagocytosis. It should be noted that many of the RPE cells observed in the course of this study were devoid of phagosomes, though receptor cell material was often in intimate contact with the apical surface. Thus the accumulation of photoreceptor debris in the subretinal space may have provided the antigen that induced a macrophagic response that went on to destroy the outer retina.

This work was supported in part by the British Retinitis Pigmentosa Society. Their generous support is acknowledged.

\section{References}

1 Kearns TP, Sayre GP. Retinitis pigmentosa, external ophthalmoplegia and complete heart block. Unusual syndrome with histologic study in one of two cases. Arch Ophthalmol 1958; 60: $280-9$.

2 Kearns TP. External ophthalmoplegia, pigmentary degeneration of the retina and cardiomyopathy: a newly recognised syndrome. Trans Am Ophthalmol Soc 1965; 63: 559-625.

3 Jager BV, Fred HL, Butler RB, Carnes WH. Occurrence of retinal pigmentation, ophthalmoplegia, ataxia, deafness and heart block. Report of a case with findings at autopsy. Am J Med 1960; 29: 888-93.

4 Daroff RB, Solitare GB, Pincus JH, Glaser GH. Spongiform encephalopathy with chronic progressive external ophthalmoplegia mimicking ocular myopathy. Neurology (Minneap) 1966; 16: 161-9.

5 Castaigne P, Lhermitte FG, Escourolle R, Chain F, Fardcau M, Hauw, JJ, Curct J, Flavigny C. Etude anatomo-clinique d'unc observation d" "ophthalmoplegia plus" avec analyse des lésions nusculares, nerveuses centrales, ocularies, myocardiques et thyroidiennes. Rev Neurol (Paris) 1977; 133: 369-86.

6 Horwitz SJ, Roessmann U. Kearns-Sayre syndrome with hypoparathyroidism. Ann Neurol 1978; 3: 513-8. 
7 Newell FW, Palascik HA. Mitochondrial disease and retinal pigmentary degeneration. In: Shimiza K, Oosterhuis J, eds. Proceedings of the 23rd International Congress of Ophthalmology. Kyoto 1978. Amsterdam: Excerpta Medica International Congress series No 450. 1979; 615-7.

8 Eagle RC, Hedges TR, Yanoff M. The atypical pigmentary retinopathy of Kearns-Sayre syndrome. A light and electron microscopic study. Ophthalmology 1982; 89: 1433-40.

9 Drachman DA. Ophthalmoplegia plus: the neurodegenerative disorders associated with progressive external ophthalmoplegia. Arch Neurol 1968; 18: 654-74.

10 Berenberg RA, Pellock JM, DiMauro S. Lumping or splitting? Ophthalmoplegia-plus or Kearns-Sayre syndrome? Ann Neurol 1977; 1: 37-54.

11 Bastiaensen LAK, Notermans SLH, Ramaekers CH. Kearns syndrome or Kearns disease. Further evidence of a genuine entity in a case with uncommon features. Ophthalmologica 1982; 184: 40-50.

12 François J. Metabolic tapetoretinal degenerations. Surv Ophthalmol 1982; 26: 293-333.

13 Bastiaensen LAK. Pigment changes in the retina in chronic progressive external ophthalmoplegia (CPEO). Acta Ophthalmol (Kbh) 1978; 56 (suppl 138): 5-36.

14 Mitsumoto H, Aprille JR, Wray SDH, Nemni R, Bradley WG. Chronic progressive external ophthalmoplegia (CPEO): clinical, morphologic and biochemical studies. Neurology (Minneap) 1983; 33: 452-61.

15 Johnson MA, Turnbull DM, Dick DJ, Sherratt HSA. A partial deficiency of cytochrome $c$ oxidase in chronic progressive external ophthalmoplegia. J Neurol Sci 1983; 60: 31-53.

16 Rieske-Nielsen E, Lou HC, Lowes M. Progressive external ophthalmoplegia. Evidence for a generalised mitochondrial disease with a defect in pyruvate metabolism. Acta Ophthalmol (Kbh) 1975; 54: 553-73.

17 Morgan-Hughes JA, Hayes DJ, Clark JB. Mitochondrial encephalomyopathies. Biochemical studies in two cases revealing defects in the respiratory chain. Brain 1982; 105: 553-82.

18 Adachi M, Torll J, Volk BW, Briet D, Wolintz A, Schneck L. Electron microscopic and enzyme histochemical studies of cerebellum, ocular and skeletal muscles in chronic progressive ophthalmoplegia with cerebellar ataxia. Acta Neuropathol (Berl) 1973; 23: 300-12.

19 Schneck L, Adachi M, Briet D, Wolintz A, Volk BW. Ophthalmoplegia plus with morphological and chemical studies of cerebellar and muscle tissues. J Neurol Sci 1973; 19: 37-44.

20 Harati Y, Patten B, Sheehan M, Judge D, Worth J. Cardiac biopsy in Kearns-Sayre syndrome. In: den Hartog Jager W, Bryn G, Heijstee A, eds. Abstracts of the Eleventh World Congress of Neurology, Amsterdam 1977. Amsterdam: Excerpta Medica International Congress series 427 1978: 318.

21 Karpati G, Carpenter S, Larbrisseau A, Lafontaine R. The kearns-Shy syndrome. A multisystem disease with mitochondrial abnormality demonstrated in skeletal muscle and skin. J Neurol Sci 1973; 19: 133-51.

22 Gonatas NK, Evangelista I, Martin J. A generalised disorder of nervous system, skeletal muscle and heart resembling Refsum's disease and Hurler's syndrome. II Ultrastructure. Am J Med 1967; 42: 169-78.

23 Brinkman CJJ, Pinckers AJLG, Broekhuyse RM. Immune reactivity to different retinal antigens in patients suffering from retinitis pigmentosa. Invest Ophthalmol Visual Sci 1980; 19: 743-50.

24 Corwin JM, Weiter JJ. Immunology of chorioretinal disorders. Surv Ophthalmol 1981; 25: 287-305.

25 Bird AC, Marshall J. Retinal receptor disorders without known metabolic abnormalities. In: Garner A, Klintworth G, eds. Pathobiology of ocular disease. New York: Dekker, 1982; 1167-220.

26 Silverstein SC, Steinman RH, Cohn ZA. Endocytosis. Ann Rev Biochem. 1977; 46: 669-722. 